\title{
El efecto de la monensina en la función productiva de vacas lecheras (Holstein)
}

\section{The effect of monensin on the productive function of Holstein dairy cows}

DOI: $10.46932 / \mathrm{sfjdv3n1-120}$

Received in: January $20^{\text {th }}, 2022$

Accepted in: February $1^{\text {st }}, 2022$

\section{Paola Isaira Correa Aguado}

Maestra en Ciencias Producción Animal en Zonas Áridas

Institution: Universidad Autónoma de Zacatecas (México)

Direccion: Jdn. Juárez \#147, Centro Historico, 98000 Zacatecas, Zac., México

Correio eletrónico: pica83@gmail.com

Fabiola Lydie Rochin Berumen

Doctorado en Gestión Educativa

Institution: Universidad Autónoma de Zacatecas (México)

Direccion: Jdn. Juárez \#147, Centro Historico, 98000 Zacatecas, Zac., México

Correio eletrónico: fabiolauaz@outlook.com

\section{Carlos Fernando Aréchiga Flores}

Doctor of Philosophy/Environmental Physiology of Reproduction

Institution: Universidad Autónoma de Zacatecas (México)

Direccion: Jdn. Juárez \#147, Centro Historico, 98000 Zacatecas, Zac., México

Correio eletrónico: arechiga.uaz@ gmail.com

\section{Romana Melba Rincón Delgado}

Doctorado en Ciencias Pecuarias

Institution: Universidad Autónoma de Zacatecas (México)

Direccion: Jdn. Juárez \#147, Centro Historico, 98000 Zacatecas, Zac., México

Correio eletrónico: rmelba47@ hotmail.com

\section{Zimri Cortés Vidauri}

Doctorado en Ciencias Agropecuarias

Institution: Universidad Autónoma de Zacatecas (México)

Direccion: Jdn. Juárez \#147, Centro Historico, 98000 Zacatecas, Zac., México Correio eletrónico: mvzzcv@ hotmail.com

\section{RESUMEN}

Se evaluó el ionóforo Monensina (MON) sobre la salud y función productiva de vacas lecheras (Holstein; $\mathrm{n}=56$ ) de un establo comercial de Aguascalientes, México. El grupo Testigo (TES) recibió la ración alimenticia y al Grupo Monensina (MON) se le agregaron $200 \mathrm{mg} /$ día de monensina en la ración, 21 días preparto a 50 días postparto. Se registraron alteraciones del periparto como Cetosis clínica (CET), Retención de Membranas Fetales (RMF), Abortos (AB), Mastitis (MAST) y otras como fiebre y neumonía. La concepción se presentó a partir del día 40 posparto. Las vacas que recibieron MON tendieron a concebir antes que las hembras del grupo testigo (TES). Se concluye que La Monensina incrementó ligeramente la eficiencia reproductiva de las vacas. No influyó en el intervalo del parto al primer servicio (IPPS), pero redujo ligeramente el intervalo del parto a la concepción (IPC). La incidencia de Cetosis y Retención de Membranas Fetales fue similar. 
Palabras clave: monensina, ionóforos, vacas lecheras, función productiva, reproduccion.

\begin{abstract}
The ionophore Monensin (MON) was evaluated on the health and productive function of dairy cows (Holstein; $\mathrm{n}=56$ ) from a commercial dairy in Aguascalientes, Mexico. The Control group (TES) received the food ration and the Monensin Group (MON) received $200 \mathrm{mg}$ /day of monensin in the ration, from 21 days prepartum to 50 days postpartum. Peripartum alterations such as clinical ketosis (CET), Retained Fetal Membranes (RMF), Abortions (AB), Mastitis (MAST) and others such as fever and pneumonia were recorded. Conception occurred from day 40 postpartum. Cows receiving MON tended to conceive earlier than females in the control group (TES). It is concluded that Monensin slightly increased the reproductive efficiency of the cows. It did not influence the interval from calving to first service (IPPS), but slightly reduced the interval from calving to conception (CPI). The incidence of Ketosis and Fetal Membrane Retention was similar.
\end{abstract}

Keywords: monensin, ionophores, dairy cows, productive function, reproduction.

\title{
1 INTRODUCCIÓN
}

Las vacas lecheras presentan un balance energético negativo (BEN) durante el periparto, es decir, consumen menos nutrientes que los necesarios para cubrir el crecimiento del feto al final de la gestación y su gasto en el inicio de la lactancia (Bell, 1995). Además, el incrementado en la producción de leche ha comprometido la función metabólica y la eficiencia reproductiva (Macmillan et al., 1996). Esto se manifiesta en el período de transición, de 3 a 4 semanas preparto a 3 a 4 semanas postparto. Bajo estas circunstancias, las vacas lecheras se ven forzadas a tomar nutrientes de sus reservas corporales, antes de cubrir sus requerimientos alrededor del parto, pero desatienden momentáneamente la reproducción.

El consumo prolongado de energía antes del parto, mayor al necesario, puede disminuir el consumo de materia seca en el período posparto (Janovick y Dracley, 2010), con sus correspondientes consecuencias: incremento del nivel de ácidos grasos no esterificados (NEFAs) y beta-hidroxibutirato (BHBA) en la circulación sanguínea y triglicéridos en hígado después del parto (Janovick et al., 2011). Por lo tanto, es preferible limitar el consumo de nutrientes antes del parto, ligeramente menor a los requeridos, para incrementar su consumo en el período posparto. Sin embargo, los resultados en este campo del conocimiento han sido contradictorios. En unos estudios se ha encontrado reducción de las alteraciones en el período posparto (Beeber, 2006), pero en otros no (Winkleman et al., 2008).

Otra opción es incrementar la disponibilidad de suplementos vitamínicos (Aréchiga et al., 1998), o glucosa en el período posparto para reducir le producción de NEFAs. Esto se puede lograr por medio de la producción de ácido propiónico, el principal precursor de glucosa hepática. Se ha encontrado que dietas complementadas con monensina incrementan la producción ruminal de ácido propiónico (Armentano y Young, 1983) y como consecuencia incrementan el metabolismo de energía en el período 
posparto (Arieli et al., 2008; Duffeld et al., 2008a, b, c). Algunos transtornos metabólicos pudieran acentuarse por las condiciones clímaticas como estrés calórico (Lozano et al., 2010).

Por lo anteror, el propósito de este trabajo de investigación fue evaluar el efecto de la suplementación alimenticia de Monensina antes del parto (Exp. 1) y antes y después del parto (Exp. 2) sobre la eficiencia metabólica, incidencia de cetosis clínica y subclínica (CET), y sobre la función reproductiva de vacas lecheras Holstein de México.

\section{MATERIALES Y MÉTODOS}

\section{Localización y Animales}

El estudio se realizó en una unidad comercial de producción de leche localizada en el municipio de Pabellón de Arteaga del estado de Aguascalientes (Establo Las Palomas). Ubicado bajo las siguientes coordenadas $-102.28^{\circ}$ de Longitud y $22.10^{\circ}$ de Latitud, a $1,885 \mathrm{~m}$ sobre el nivel del mar (snm). El estudio incluyó vacas Holstein en plena lactancia $(n=218)$. Las vacas fueron sometidas al manejo de alimentación y reproducción de rutina del establo.

\section{Tratamientos:}

\section{Experimento 1: Monensina Preparto.}

El estudio incluyó vacas Holstein en plena lactancia $(n=162)$, con buena condición corporal y clínicamente sanas, las cuales fueron seleccionadas y repartidas aleatoriamente en dos grupos:
1) Grupo Testigo (TEST)
2) Grupo Monensina (MON; i.e., Rumensin ${ }^{2}$, Elanco, Greenfield, MS)

Las vacas en el grupo tratado recibieron un bolo vía oral conteniendo $32 \mathrm{~g}$ de monensina sódica (Rumensin bolos $\mathrm{ABC}^{\circledR}$, Elanco, Greenfield, MS, USA) aproximadamente $25 \mathrm{~d}$ antes de la fecha esperada de parto (i.e., $255 \pm 3$ d de gestación).

\section{Experimento 2: Monensina Pre y Post-Parto.}

Las vacas lecheras $(n=56)$ fueron divididas al azar en dos grupos:

1) Grupo Testigo (TEST), recibiendo la ración totalmente mezclada (RTM) del establo.

2) Grupo Monensina (MON), recibiendo además de la ración totalmente mezclada del establo (RTM), una suplementación adicional de $200 \mathrm{mg} / \mathrm{d}$ de Monensina (Rumensin® Elanco México, Guadalajara, Jalisco, México) durante el periodo comprendido entre el día 21 antes de la fecha esperada de parto hasta el día 50 postparto (PP) (i.e., aproximadamente $70 \mathrm{~d}$ ). 


\section{Variables de respuesta}

Se evaluó el efecto del tratamiento sobre la incidencia de cetosis (CET; clínica y subclínica) utilizando tiras reactivas $\left(\right.$ keto test $\left.^{\circledR}\right)$ para el diagnóstico. Además, sobre la función reproductiva (IPPS; IPC y NSC) y sobre las causas de desechos de las vacas Holstein.

\section{Análisis Estadístico}

\section{Experimento 1}

Se utilizó el procedimiento PROC FREQ de SAS para establecer los efectos de asociación entre tratamiento*cetosis, tratamiento*estado reproductivo, mediante Chi-cuadrada o prueba exacta de Fisher dependiendo del caso. Además, se utilizó el PROC GLM para evaluar los efectos principales del tratamiento, cetosis y número de lactancias y se obtuvieron las medias ajustadas (opción LSMEANS) y el error estándar (opción STDERR).

\section{Experimento 2}

Los datos se analizaron con R (R. Core Team, 2017). Se trabajó con las variables cualitativas por medio de pruebas de bondad que se ajustaron para tablas de contingencia por medio de $\mathrm{X}$ cuadrada de Pearson y la prueba exacta de Fisher.

Para el análisis de supervivencia se utilizó el paquete de R Survival (Therneau, 2015), se utilizaron modelos de Kaplan y Meider para determinar el tiempo de llegada de concepción y el primer servicio, para ello se utilizó la función Survfit (). Se utilizó ña función Surv () para crear los objetos de supervivencia. También se utilizaron pruebas de supervivencia entre tratamientos con la función Survdiff (), se realizó una regresión por medio de la función Surveg () basada en la distribución N de Weibull para el tiempo al primer servicio y de tiempo de concepción (Terry et al.2000).

\section{RESULTADOS}

Los resultados obtenidos del presente estudio los podemos enumerar de acuerdo a cada experimento:

\section{Experimento 1}

\section{Incidencia de Cetosis:}

La administración oral de un bolo conteniendo $32 \mathrm{~g}$ de monensina sódica a los $255 \pm 3 \mathrm{~d}$ de gestación ( 25 d pre-parto) redujo la incidencia de cetosis clínica en un $22 \%$. En el grupo de vacas suplementadas con monensina sódica el $65 \%$ no presentó cetosis, mientras que en el grupo testigo, solamente el $43.03 \%$ evitó la incidencia de cetosis $(\mathrm{P}<0.002)$. 
La incidencia de cetosis clínica se redujo de $39.2 \%$ en el grupo testigo a $14.8 \%$ en el grupo suplementado con monensina sódica (i.e., 24.4\%). Sin embargo, contrario a lo esperado, la incidencia de cetosis subclínica fue muy similar (testigo $=17.7 \%$ vs. Monensina $=19.75 \% ; \mathrm{P}<0.002$ ).

\section{Comportamiento Reproductivo:}

Las vacas tratadas con monensina sódica presentaron un mayor porcentaje de preñez que las vacas en el grupo testigo (63.33 vs. $47.06 \%$ de preñez) y disminuyó el porcentaje de vacas no gestantes (6.7 vs. $8.8 \%)$.

Así mismo, la monensina sódica no afectó a otros parámetros reproductivos como el No. de servicios por concepción (NSC) ó el intervalo parto a concepción (IPC) $(\mathrm{P}<0.05)$. Sin embargo, si incrementó en 26 días, el intervalo parto a primer servicio (IPPS) de las vacas en plena lactancia (Testigo=50.71 vs. Monensina=76.76 d; $\mathrm{P}<0.05$ ).

Cuadro 1. Efecto de la Monensina sódica sobre la incidencia de cetosis en vacas Holstein.

\begin{tabular}{|l|c|c|c|}
\hline \multicolumn{1}{|c|}{ Alteración } & Testigo & $\begin{array}{c}\text { Monensina Sódica } \\
\left.\text { (Rumensin }{ }^{1}\right)\end{array}$ & Total \\
\hline Ausencia de Cetosis & $43.03 \%(34 / 79)$ & $65.43 \%(53 / 81)$ & $87 / 160$ \\
\hline Cetosis Subclínica & $17.72 \%(14 / 79)$ & $19.75 \%(16 / 81)$ & $30 / 160$ \\
\hline Cetosis Clínica & $39.24 \%(31 / 79)$ & $14.81 \%(12 / 81)$ & $43 / 160$ \\
\hline Porcentaje Total & $100.00 \%(79 / 79)$ & $100.00 \%(81 / 81)$ & $100 \%(160 / 160)$ \\
\hline
\end{tabular}

${ }^{1}$ El efecto del tratamiento con monensina sódica presenta diferencias estadísticamente significativas $(\mathrm{P}<0.002)$ determinado por las pruebas de Chi-cuadrada y Prueba exacta de Fisher.

Cuadro 2. Efecto de la Monensina sódica, la incidencia de cetosis, y el número de lactancias sobre los parámetros reproductivos.

\begin{tabular}{|l|c|c|c|}
\hline & $\begin{array}{c}\text { No. de Servicios por } \\
\text { Concepción }\end{array}$ & $\begin{array}{c}\text { Intervalo Parto a } \\
\text { Primer Servicio }\end{array}$ & $\begin{array}{c}\text { Intervalo Parto a } \\
\text { Concepción }\end{array}$ \\
\hline Tratamiento ${ }^{\mathbf{1}}$ & & & $68.21 \pm 15.24$ \\
\hline Testigo Sódica & $2.20 \pm 0.36$ & $50.71 \pm 4.84$ & $72.78 \pm 13.77$ \\
\hline $\begin{array}{l}\text { Monensina } \\
\text { Rumensin®) }\end{array}$ & $2.33 \pm 0.36$ & $76.76 \pm 4.80^{2}$ & \\
\hline No. de Lactancias & & & $65.34 \pm 15.20$ \\
\hline \multicolumn{1}{|c|}{$\geq$} & $2.26 \pm 0.37$ & $61.15 \pm 5.11$ & $72.38 \pm 19.39$ \\
\hline 3 & $1.77 \pm 0.49$ & $62.47 \pm 6.52$ & $73.77 \pm 15.90$ \\
\hline 3 & $2.75 \pm 0.39$ & $67.59 \pm 5.35$ & $83.81 \pm 8.64$ \\
\hline Cetosis ${ }^{3}$ & & & $78.73 \pm 20.17$ \\
\hline Ausencia de Cetosis & $2.65 \pm 0.25$ & $66.06 \pm 3.47$ & $48.94 \pm 29.48$ \\
\hline Cetosis Subclínica & $1.32 \pm 0.70$ & $59.45 \pm 9.59$ & \\
\hline Cetosis Clínica & $2.81 \pm 0.45$ & $65.70 \pm 5.89$ & \\
\hline
\end{tabular}

${ }^{1}$ Diferencia estadísticamente significativa $(\mathrm{P}<0.05)$.

${ }^{2}$ Diferencia estadística altamente significativa $(\mathrm{P}<0.0001)$.

${ }^{3}$ No existe diferencia estadísticamente significativa (N.S. $\left.=\mathrm{P}>0.05\right)$. 
Cuadro 3. Efecto de la Monensina sódica sobre el estado gestacional de vacas Holstein lactantes.

\begin{tabular}{|l|c|cc|cc|}
\hline & \multicolumn{2}{|c|}{ Testigo } & \multicolumn{2}{|c|}{$\begin{array}{c}\text { Monensina sódica } \\
\text { (Rumensin®) }\end{array}$} & \multicolumn{2}{c|}{ TOTAL } \\
\hline Vacas Inseminadas & $44.12 \%(15 / 34)$ & $30.0 \%$ & $(9 / 30)$ & $37.5 \%$ & $(24 / 64)$ \\
\hline Vacas Gestantes & $47.06 \%(16 / 34)$ & $63.33 \%$ & $(19 / 30)$ & $54.69 \%$ & $(35 / 64)$ \\
\hline $\begin{array}{l}\text { Vacas No Gestantes } \\
\text { vacias) }\end{array}$ & $8.82 \%(3 / 34)$ & $6.67 \%$ & $(2 / 30)$ & $7.81 \%$ & $(5 / 64)$ \\
\hline TOTAL & $53.12 \%(34 / 64)$ & $46.88 \%$ & $(30 / 64)$ & $100 \%$ & $(64 / 64)$ \\
\hline
\end{tabular}

${ }^{1} \mathrm{El}$ efecto del tratamiento con monensina sódica presenta diferencias estadísticamente significativas $(\mathrm{P}<0.05)$ determinado por la Prueba exacta de Fisher.

Cuadro 4. Efecto de la Monensina sódica sobre la tasa de eliminación.

\begin{tabular}{|c|c|c|c|}
\hline & Testigo & $\begin{array}{l}\text { Monensina sódica }^{1} \\
\text { (Rumensin } 囚)\end{array}$ & TOTAL \\
\hline $\begin{array}{c}\text { Vacas } \\
\text { Producción }\end{array}$ & $50.8 \% \quad(33 / 65)$ & $49.2 \% \quad(32 / 65)$ & $100 \% \quad(65 / 65)$ \\
\hline $\begin{array}{rr}\begin{array}{r}\text { Vacas } \\
\text { (desecho) }\end{array} & \text { Eliminadas } \\
\end{array}$ & $66.7 \%$ & $33.3 \% \quad(1 / 3)$ & $100 \%(3 / 3)$ \\
\hline TOTAL & $51.47 \% \quad(35 / 68)$ & $48.53 \% \quad(33 / 68)$ & $100 \% \quad(68 / 68)$ \\
\hline
\end{tabular}

${ }^{1}$ No existió diferencia estadísticamente significativa.

\section{Experimento 2}

En la Figura 1, podemos observar una disminución en los casos de cetosis (CET) en respuesta a la suplementación con Monensina -21 d a 50 d con relación al parto (Tratamiento 2).

En la Figura 2, podemos observar que en el grupo de vacas Holstein que recibió Monensina (MON), los porcentajes de vacas gestantes fueron muy similares (Tratamiento 2).

En la Figura 3, podemos observar que el número de vacas que presentaron al parto la retención de la placenta ó la retención de membranas fetales (RMF), no presentó una diferencia estadísticamente significativa, Tratamiento 2 .

En la Figura 4, se presenta el intervalo entre el parto y la inseminación artificial. No se presentaron variaciones en esta variable de acuerdo al tratamiento. Algunas vacas reanudaron la manifestación de los estros a los 30 días después del parto, mientras que otras lo hicieron más tardíamente, hasta 80 días durante el período posparto.

En la Figura 5, aparece el intervalo entre parto y el porcentaje de concepción. Las vacas con MON (grupo tratado) tendieron a concebir antes que las hembras del grupo testigo. El 50\% de las vacas del grupo tratado concibieron en el trascurso de 120 días del período posparto. La concepción se presentó a partir del día 40 posparto.

Las vacas con menor intervalo entre el parto y la inseminación artificial, también redujeron el intervalo entre el parto y la concepción.

El intervalo entre parto e inseminación artificial tendió a reducirse conforme se incrementaron los partos. 


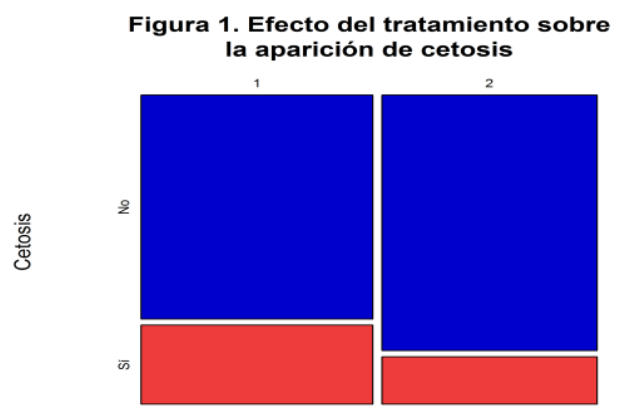

Tratamiento
Figura 2. Efecto del tratamiento sobre el estado reproductivo

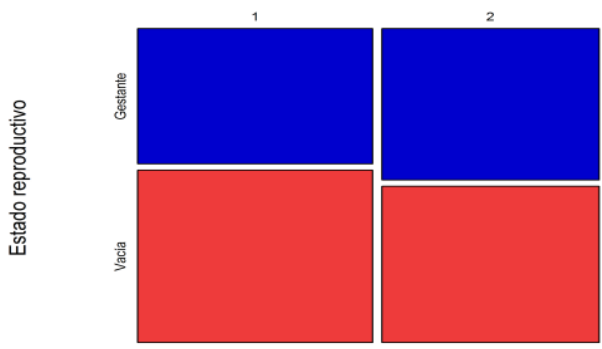

Tratamiento

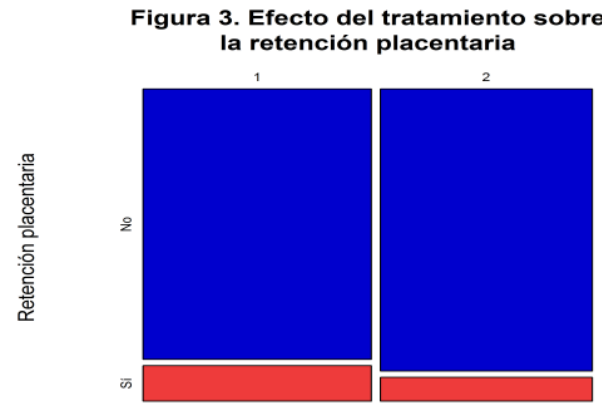

Tratamiento

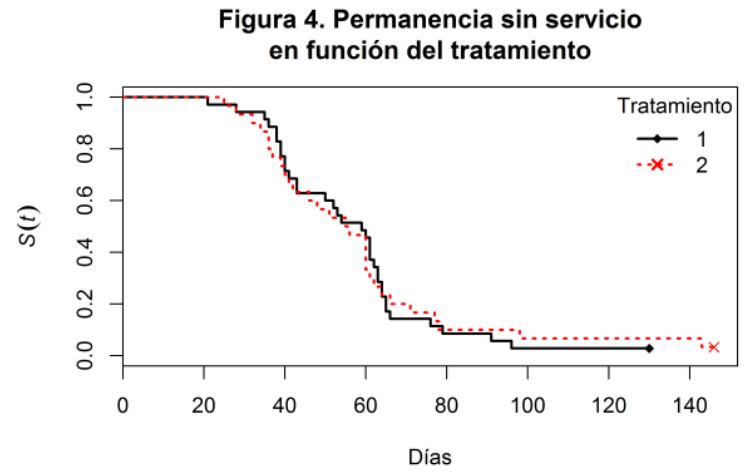

Figura 5. Días abiertos en función del tratamiento

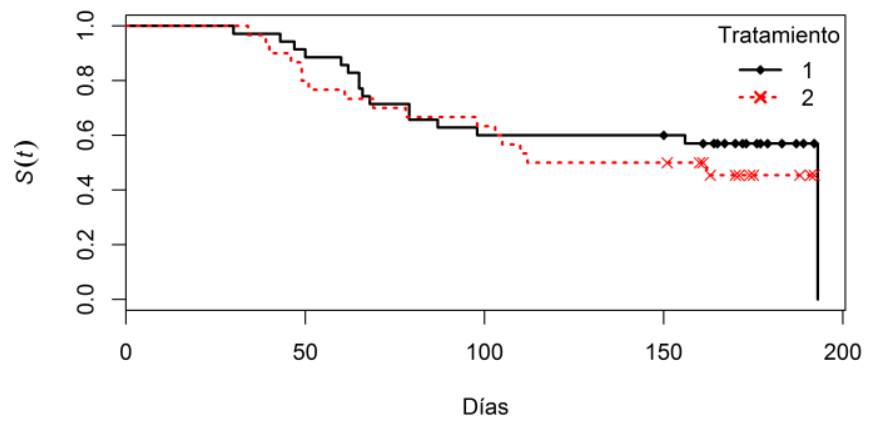




\section{CONCLUSIONES Y RECOMENDACIONES}

\section{Efecto de la Monensina en la Incidencia de Cetosis}

La suplementación con un bolo conteniendo $32 \mathrm{~g}$ del ionóforo monensina sódica a partir de aproximadamente un mes antes del parto de las vacas lecheras (Holstein), redujo significativamente la incidencia de Cetosis (CET) 22\% en comparación al grupo Testigo (i.e., 35\% vs. 57\%) considerando las presentaciones clínicas y subcinicas. En el cas de la Cetosis Clínica la incidencia fue de $\mathrm{MON}=14.8 \%$ vs. TEST $=39.2 \%$. Sin embargo, en el caso de la Cetosis Subclínica la incidencia fue muy similar entre grupos $(\mathrm{MON}=19.7$ vs. $\mathrm{TEST}=17.7 \%)$

\section{Efecto de la Monensina en la Función Reproductiva}

La suplementación con Monensina mejoró los porcentajes de preñez de las vacas lecheras testigo (63.33 vs. $47.06 \%$ de preñez) y disminuyó el porcentaje de vacas no gestantes (6.7 vs. 8.8\%). Sin embargo, incrementó el intervalo de parto al primer servicio (i.e., primera inseminación; Monensina=76.76 d vs. Testigo=50.71; $\mathrm{P}<0.05$ ). Sin embargo, no se comprometieron parámetros reproductivos como el número de servicios por concepción (NSC), ó el intervalo del parto a la concepción (IPC), los cuales fueron muy similares entre grupos MON y TEST.

Cuando se amplió el periodo de suplementación de la Monensina del día -21 antes del parto hasta el día 50 postparto (Experimento 2), las vacas tratadas con Monensina tendieron a reducir la incidencia de cetosis clínica (15.6 vs. 26.1\%), y de retención de membranas fetales (7.8 vs. 11.6\%). Se incrementó ligeramente la eficiencia reproductiva de las vacas, pero no influyó en el intervalo del parto al primer servicio. Sin embargo el intervalo del parto a la concepción (IPC) se redujo ligeramente.

El presente trabajo sugiere un efecto positivo de la Monensina en mejorar la función metabólica reduciendo problemas como la incidencia de cetosis (CET), la incidencia de retención de membranas fetales (RMF) y mejorando algunos parámetros reproductivos (IPC). Sin embargo, es necesario continuar investigando el efecto del ionóforo Monensina sobre otras alteraciones del periparto y la función productiva y reproductiva de las vacas lecheras Holstein de México. 


\section{REFERENCIAS}

Arieli A, Dicken U, Dagoni I, Spirer Y, Zamwel S. 2008. Production and health of cows given monensin prepartum and a high-energy diet postpartum. J Dairy Sci 91: 1845-1851.

Aréchiga CF, Staples CR, McDowell LR, Hansen PJ. 1998. Effects of timed Insemination and supplemental $\beta$-carotene on Reproduction and Milk Yield of Dairy Cows Under Heat Stress. Journal of Dairy Science 81:390-402.

Armentano LE, Yung JW. 1983. Production and metabolism of volatile fatty acids, glucose and $\mathrm{CO}_{2}$ in steers and the effect of monensin on volatile fatty acid kinetics. J Nutr 91: 1845-1851.

Beeber DE. 2006. The impact of controlled nutrition during the dry period on dairy cow health, fertility and performance. Anim Reprod Sci 96: 212-226.

Bell AW. 1995. Regulation of organic nutrient metabolism during transition from late pregnancy to early lactation. J Anim Sci 73: 2804-2819.

Duffield TF, Rabiee AR, Lean IJ. 2008a. A meta-analysis of the impact of monensin in lactating dairy cattle. Part 1. Metabolic effects. J Dairy Sci 91: 1334-1346.

Duffield TF, Rabiee AR, Lean IJ. 2008b. A meta-analysis of the impact of monensin in lactating dairy cattle. Part 2. Production effects. J Dairy Sci 91: 1347-1360.

Duffield TF, Rabiee AR, Lean IJ. 2008c. A meta-analysis of the impact of monensin in lactating dairy cattle. Part 3. Health and reproduction. J Dairy Sci 91: 2328-2341.

Janovick NA, Boisclair YR, Drackely JK. 2011. Prepartum dietary energy intake affects metabolism and health during the periparturient period in primiparous and multiparous Holstein cows. J Dairy Sci 94: 1385-1400.

Janovick NA, Drackley JK. 2010. Prepartum dietary management of energy intake affects postpartum intake and lactation performance by primiparous and multiparous Holstein cows. J Dairy Sci, 93: 3086-3102.

Lozano-Domínguez RR, Asprón-Pelayo MA，Vásquez-Peláez CG，González-Padilla E，Aréchiga-Flores CF. 2010. Efecto del estrés calórico sobre la producción embrionaria en vacas superovuladas y la tasa de gestación en receptoras. Rev. Mex. De Cienc. Pecuarias 1(3): 189-203. ISSN 2007-1124.

Macmillan KLS, Lean IJ, Westwood CT. 1996. The effects of lactation on the fertility of dairy cows. Australian Vet J73:141-147.

R. Core Team, 2017. A language and environment for statistical computing. R Foundation for Statistical Computing, Vienna, Austria. http://www.R-project.org

SAS Institute Inc. 2015. SAS/IML® 14.1 User's Guide. Cary, NC: SAS Institute Inc

Terry M, Therneau, Patricia M Grambsch. 2000. Modeling Survival Data: Extending the Cox Model. Springer, New York. \pm SBN0-387-98784-3. 2000.

Therneau T. 2015. A Package for Survival Analysis in S. version 2.38. 2015.

Winkleman LA, Elsasser TH, Reynolds CK. 2008. Limit-feeding a high-energy diet to meet energy requirements in the dry period alters plasma metabolite concentrations but does not affect intake or milk production in early lactation. J Dairy Sci 91:1067-1079. 